\title{
CONTINUOUS FUNCTIONS ON MULTIPOLAR SETS
}

\author{
RAMASAMY JESURAJ
}

\begin{abstract}
Let $\Omega=\Omega_{1} \times \cdots \times \Omega_{n}(n>1)$ be a product of $n$ Brelot harmonic spaces each of which has a bounded potential, and let $K$ be a compact subset of $\Omega$. Then, $K$ is an $n$-polar set with the property that every $i$-section $(1 \leqslant i<n)$ of $K$ through any point in $\Omega$ is $(n-i)$ polar if and only if every positive continuous function on $K$ can be extended to a continuous potential on $\Omega$. Further, it has been shown that if $f$ is a nonnegative continuus function on $\Omega$ with compact support, then $M R f$, the multireduced function of $f$ over $\Omega$, is also a continuous function on $\Omega$.
\end{abstract}

1. Introduction. Let $\Omega_{j}$ for $j=1,2, \ldots, n$ be locally compact spaces with countable basis for the topology and be Brelot spaces [5]. The principal results of this paper (cf. Theorem 3 and Theorem 12) characterize certain exceptional compact sets $K$ contained in the product space $\Omega_{1} \times \Omega_{2} \times \cdots \times \Omega_{n}$ in terms of extendibility of positive continuous functions on $K$, to a positive $n$-potential on the entire space. This is a natural generalization of the corresponding results in [7] in the case of a single harmonic space. A key result, independent of interest, needed in the proof of the main results in Theorem 5 shows that the multireduced function of a continuous function is continuous. The results of this paper formed a part of my $\mathrm{Ph}$. D. thesis submitted to McGill University [6]. I would like to thank Professor Gowrisankaran for his help in preparation of this work.

We shall use the notation and results from [3, 4 and 8] concerning $n$-harmonic, $n$-superharmonic functions, and $n$-potentials. We assume that there is a bounded $n$-superharmonic function on $\Omega_{1} \times \cdots \times \Omega_{n}$. Notice that it is equivalent to the assumption that each $\Omega_{j}(j=1, \ldots, n)$ has a bounded potential. If constants are superharmonic, then this assumption trivially holds. Throughout this paper, unless it is explicitly mentioned, $n$ is an integer $\geqslant 2$, and we denote $\Omega_{1} \times \cdots \times \Omega_{n}$ by $\Omega$.

In the course of proving our main results we need a number of results that are routine generalizations of similar ones in the single variable case. The proofs of some of them are not quite obvious, and can be found in [6].

Definition 1. Let $f$ be an extended real-valued function on $\Omega$. Define $M R f(x)$ $=\inf \{u(x): u$ is $n$-hyperharmonic, and $u \geqslant f$ on $\Omega\}$. For each subset $E$ of $\Omega$, let $X_{E}$ be the characteristic function of $E$, and let $X_{E} \cdot f$ be the pointwise product function on $\Omega$. The function $\operatorname{MR}\left(X_{E} \cdot f\right)$ is called the multireduced function of $f$ over $E$.

Received by the editors September 11, 1984 and, in revised form, November 1, 1985. 1980 Mathematics Subject Classification (1985 Revision). Primary 31D05. 
Observe that $\left(M R\left(X_{E} \cdot f\right)\right)^{\uparrow}$, the lower semicontinuous regularization (see [1]) of $\operatorname{MR}\left(X_{E} \cdot f\right)$, is an $n$-hyperharmonic function on $\Omega$.

Let us recall the definition of a section from [8].

Definition 2. Let $E$ be a subset of $\Omega, n>1$, and $i$ an integer such that $1 \leqslant i \leqslant n-1$. Let $r_{1}, \ldots, r_{i}$ be integers with $1 \leqslant r_{1}<r_{2}<\cdots<r_{i} \leqslant n$. Let $t=$ $\left(x_{s_{1}}, \ldots, x_{s_{j}}\right)$ be a point in $\Omega_{s_{1}} \times \cdots \times \Omega_{s_{j}}(1 \leqslant j \leqslant n)$ with $\left\{r_{1}, \ldots, r_{i}\right\} \subseteq$ $\left\{s_{1}, \ldots, s_{j}\right\} \subseteq\{1,2, \ldots, n\}$. Then the $\left(r_{1}, \ldots, r_{i}\right)$-section of $E$ through $t$ is defined as

$z$ in $\prod_{k=1}^{n} \Omega_{k}, \quad\left(y_{1}, \ldots, y_{n}\right)$ is in $E$, whenever $y_{k}=x_{k}$ for $k$ in $\left\{r_{1}, \ldots, r_{i}\right\}$,

$k$ not in $\left\{r_{1}, \ldots, r_{i}\right\}, \quad y_{k}=z_{k}$ for $k$ not in $\left\{r_{1}, \ldots, r_{i}\right\}$,

which would be denoted by $E\left[\left(r_{1}, \ldots, r_{i}\right), t\right]$. For $x$ in $\Omega$, an $i$-section of $E$ through $x$ is always denoted by $E\left[\left(r_{1}, \ldots, r_{i}\right), x\right]$ for some $\left(r_{1}, \ldots, r_{i}\right)$.

2. A characterization of a class of multipolar sets. The following theorem gives a necessary condition for a compact set to be $n$-polar. We recall that a set $E$ is $n$-polar if there exists a positive $n$-superharmonic function which is identically infinity on $E$.

THEOREM 3. Let $K$ be a compact subset of $\Omega$ such that every positive continuous function on $K$ can be uniformly approximated on $K$ by positive $n$-superharmonic functions on $\Omega$. Then,

(1) If $K$ has more than one point, then $K$ is an $n$-polar set.

(2) In addition, if point sets are polar in $\Omega_{j}$ for $j=1,2, \ldots, n$, then

(a) Every $i$-section of $K$ through any point in $\Omega$ is $(n-i)$-polar for $i=1,2, \ldots, n-$ 1.

(b) Given $z$ in $\Omega$ such that $z$ not in $K$, there is a positive $n$-superharmonic function $u$ on $\Omega$ such that $u(z)<\infty$ and $u(x)=\infty$ for all $x$ in $K$.

Proof. (1) The proof of the fact that the hypothesis implies the $n$-polarity of $K$ is very similar to the proof of Theorem 1 of [7].

(2.a) This part is proved by induction on $n$. Let us make the induction assumption that for all products of Brelot spaces $\Omega_{1} \times \cdots \times \Omega_{m}$ with $m<n$, the hypothesis of the theorem implies the property (a).

Let us now consider a compact set $K$ contained in $\Omega$ satisfying the hypothesis. Fix $z$ in $\Omega$, and $i$ such that $1 \leqslant i \leqslant n-1$. By a suitable rearrangement, if necessary, we may consider the $i$-section $K[(1,2, \ldots, i), z]$ to be the general $i$-section of $K$. This set is compact and contained in $\Omega_{i+1} \times \cdots \times \Omega_{n}$. Let us consider further the nontrivial case when $K[(1,2, \ldots, i), z]$ contains more than one point. We shall show that this set is $(n-i)$-polar. By the induction hypothesis, it suffices to prove that an arbitrary positive continuous function $f$ on $K[(1,2, \ldots, i), z]$ can be uniformly approximated on $K[(1,2, \ldots, i), z]$ by positive $(n-i)$-superharmonic functions.

Let us consider the copy $L[i, z]$ of the above compact set in $K$, viz., $L[i, z]=$ $\left\{\left(z_{1}, \ldots, z_{i}, y_{i+1}, \ldots, y_{n}\right)\right.$ in $\left.K\right\} . L[i, z]$ is a compact subset of $K$, and $f$ can be considered as a positive continuous function on this set. By the Tietze extension theorem, there exists a positive continuous function $g$ on $K$ which extends $f$. By 
hypothesis, for $\varepsilon>0$, there exists a positive $n$-superharmonic function $u$ on $\Omega$ such that $|u-g|<\varepsilon$ on $K$ uniformly. Set

$$
v\left(y_{i+1}, \ldots, y_{n}\right)=u\left(z_{1}, \ldots, z_{i}, y_{i+1}, \ldots, y_{n}\right) \text {. }
$$

This function is $(n-i)$ superharmonic on $\Omega_{i+1} \times \cdots \times \Omega_{n}$, and clearly $|f-v|$ $<\varepsilon$ uniformly on $K[(1,2, \ldots, i), z]$. Hence, by the induction assumption we conclude that $K[(1,2, \ldots, i), z]$ is $(n-i)$-polar. This concludes the proof of (2.a).

(2.b) By Theorem 2.4 of [8], for $n$-polar sets, the properties (2.a) and (2.b) are equivalent. This completes the proof of the theorem.

Using the results of [3], it is easy to prove the following proposition.

Proposition 4. Let $v$ be an $n$-superharmonic function on $\Omega$, and let $\delta_{i}$ be a regular domain in $\Omega_{i}, i=1,2, \ldots, n$. For $j=0,1,2, \ldots, n$, define $v_{j}$ on $\Omega$ as follows.

$$
v_{0}\left(x_{1}, \ldots, x_{n}\right)=v\left(x_{1}, \ldots, x_{n}\right) \text {, }
$$

and for $j=1,2, \ldots, n$, let

$$
v_{j}^{\prime}\left(x_{1}, \ldots, x_{n}\right)=\left\{\begin{array}{l}
\int v_{j-1}\left(x_{1}, \ldots, x_{j-1}, z, x_{j+1}, \ldots, x_{n}\right) d \rho_{x_{j}}^{\delta_{j}}(z) \quad \text { if } x_{j} \text { in } \delta_{j}, \\
v_{j-1}\left(x_{1}, \ldots, x_{n}\right) \quad \text { if } x_{j} \text { not in } \delta_{j} .
\end{array}\right.
$$

Then,

(1) $v_{j}$ is an $n$-superharmonic function on $\Omega$, and $v_{j}(x) \leqslant v_{j-1}(x)$ for all $x$ in $\Omega$.

(2) $v_{n}$ is an $n$-harmonic function on $\delta_{1} \times \cdots \times \delta_{n}$.

(3) $v_{n}(x)=v(x)$ for every $x$ in $\left(\Omega_{1} \backslash \delta_{1}\right) \times \cdots \times\left(\Omega_{n} \backslash \delta_{n}\right)$.

The following theorem is a generalization, to product of harmonic spaces, of a similar result in a single harmonic space (see Proposition 2.2.3 of [2]).

THEOREM 5. If $f$ is a nonnegative continuous function on $\Omega$ with compact support, then $M R f$ is a continuous function on $\Omega$.

Recall that the support of a real-valued function is the smallest closed set outside which the function is identically zero.

To prove this theorem we need several lemmas.

LEMMA 6. Let $I$ be an indexing set, and $\left\{g_{i}: i\right.$ in $\left.I\right\}$ a family of continuous functions on $\Omega$ with compact supports. Let there be an $\varepsilon>0$ such that $\left|g_{i}(x)-g_{j}(x)\right| \leqslant \varepsilon$ for all $x$ in $\Omega$, and for all $i$ and $j$ in $I$. Further, let us suppose that one of the following two holds:

(1) The constant function 1 is n-superharmonic on $\Omega$.

(2) There is a relatively compact open set $U$ of $\Omega$ such that support of $g_{i}$ is contained in $U$ for every $i$ in $I$.

Then, there is a constant $c>0$ such that $\left|M R g_{i}(x)-M R g_{j}(x)\right|<c \varepsilon$ for $x$ in $\Omega$, and for $i$ and $j$ in I. In case (1) holds, $c$ can be chosen to be 1, and in the case where (2) holds, $c$ depends only upon $U$ (not on the functions $g_{i}$ ).

Proof. If condition (1) holds the proof is trivial. Suppose that (2) holds. Choose a nonnegative continuous function $f$ on $\Omega$ with compact support and $f=1$ on $U$. Then, $M R f$ is a bounded $n$-potential on $\Omega$, and hence there is a $c>0$ such that 
$M R f \leqslant c$ on $\Omega$. Observing the fact that $g_{i} \leqslant g_{j}+\varepsilon f$ on $\Omega$ for $i$ and $j$, the result follows, and the proof is complete.

LEMMA 7. Let $f$ be a continuous function on $\Omega$ with compact support. Let $1 \leqslant j<n$ be fixed. Further, let $d$ be a metric on $\Omega_{1} \times \cdots \times \Omega_{j}$. For $x^{\prime}$ in $\Omega_{1} \times \cdots \times \Omega_{j}$, define $f_{x^{\prime}}$ on $\Omega_{j+1} \times \cdots \times \Omega_{n}$ as $x^{\prime \prime} \mapsto f\left(x^{\prime}, x^{\prime \prime}\right)$. Then given $\varepsilon>0$, there is an $\eta>0$ such that $\left|M R f_{x^{\prime}}(z)-M R f_{y^{\prime}}(z)\right|<\varepsilon$, for every $z$ in $\Omega_{j+1} \times \cdots \times \Omega_{n}$ and for all $x^{\prime}, y^{\prime}$ in $\Omega_{1} \times \cdots \times \Omega_{j}$, with $d\left(x^{\prime}, y^{\prime}\right)<\eta$. (Note that the multireduced functions $M R f_{x^{\prime}}$ and $M R f_{y^{\prime}}$ are defined with respect to the space $\Omega_{j+1} \times \cdots \times \Omega_{n}$.)

Proof of Theorem 5. The proof is by induction on $n$. For $n=1$, the result is true (see Proposition 2.2.3 of [2]). Let us prove the result for the case $n=2$. Set $v=M R f$. Then, it is obvious that $v$ is lower semicontinuous on $\Omega$. Therefore, it suffices to prove the upper semicontinuity of $v$ by proving that for any $\left(z_{1}, z_{2}\right)$ in $\Omega_{1} \times \Omega_{2}$

$$
\limsup _{\substack{\left(x, x_{2}\right) \rightarrow\left(z_{1}, z_{2}\right) \\\left(x_{1}, x_{2}\right) \text { in } \Omega_{1} \times \Omega_{2}}} v\left(x_{1}, x_{2}\right) \leqslant v\left(z_{1}, z_{2}\right) .
$$

Let $\left(z_{1}, z_{2}\right)$ in $\Omega_{1} \times \Omega_{2}$ be fixed, and let $\varepsilon>0$. Since $f$ is continuous, and $v$ is lower semicontinuous, there are relatively compact open neighborhoods $U_{1}$ and $U_{2}$ of $\left(z_{1}, z_{2}\right)$ with $\bar{U}_{1} \subset U_{2}$, and there is a 2-harmonic function $h$ on $\Omega_{1} \times \Omega_{2}$ satisfying the following conditions.

1. $h\left(z_{1}, z_{2}\right)=1$.

2. For all $\left(x_{1}, x_{2}\right)$ in $\bar{U}_{1} \subset U_{2}$, we have

$$
\begin{aligned}
& h\left(x_{1}, x_{2}\right) \geqslant 1-\varepsilon, \\
& f\left(x_{1}, x_{2}\right) \leqslant\left(f\left(z_{1}, z_{2}\right)+\varepsilon\right) h\left(x_{1}, x_{2}\right),
\end{aligned}
$$

and

$$
v\left(x_{1}, x_{2}\right) \geqslant\left(v\left(z_{1}, z_{2}\right)-\varepsilon\right) h\left(x_{1}, x_{2}\right)
$$

Let $d_{i}$ be a metric in $\Omega_{i}(i=1,2)$. Then, by Lemma 7 , there is an $\eta>0$ such that, $R f_{x}(s)-\varepsilon<R f_{y}(s)<R f_{x}(s)+\varepsilon$ for all $s$ in $\Omega_{1}$, and

$$
\begin{aligned}
R f_{x}(t)-\varepsilon<R f_{y}(t)<R f_{x}(t)+\varepsilon & \text { for all } t \text { in } \Omega_{2}, \\
& \text { if } d_{1}\left(x_{1}, y_{1}\right)<\eta \text {, and } d_{2}\left(x_{2}, y_{2}\right)<\eta
\end{aligned}
$$

Now, choose $\delta_{i}$, a regular domain in $\Omega_{i}, i=1,2$, such that $\left(z_{1}, z_{2}\right)$ is in $\delta_{1} \times \delta_{2} \subset \bar{\delta}_{1} \times \bar{\delta}_{2} \subset U_{2}$. We may assume that the diameter of $\delta_{i}$ is small enough and it satisfies

$$
\int d \rho_{x_{i}}^{\delta_{i}}(t) \geqslant 1-\varepsilon \text { for every } x_{i} \text { in } \delta_{i}, i=1,2
$$


Put $w=v+2 h$. Then, $w$ is a 2-superharmonic function on $\Omega_{1} \times \Omega_{2}$. Define a function $u$ as follows.

$$
u\left(x_{1}, x_{2}\right)= \begin{cases}\iint w\left(y_{1}, y_{2}\right) d \rho_{x_{1}}^{\delta_{1}}\left(y_{1}\right) d \rho_{x_{2}}^{\delta_{2}}\left(y_{2}\right) & \text { if }\left(x_{1}, x_{2}\right) \text { in } \delta_{1} \times \delta_{2}, \\ \int w\left(x_{1}, y_{2}\right) d \rho_{x_{2}}^{\delta_{2}}\left(y_{2}\right) & \text { if } x_{1} \text { not in } \delta_{1}, \text { and } x_{2} \text { in } \delta_{2}, \\ \int w\left(y_{1}, x_{2}\right) d \rho_{x_{1}}^{\delta_{1}}\left(y_{1}\right) & \text { if } x_{1} \text { in } \delta_{1}, \text { and } x_{2} \text { not in } \delta_{2}, \\ w\left(x_{1}, x_{2}\right) & \text { otherwise. }\end{cases}
$$

It is clear that $u$ is a 2-superharmonic function on $\Omega_{1} \times \Omega_{2}, u \leqslant w$, and $u$ is 2-harmonic on $\delta_{1} \times \delta_{2}$.

We claim that $u \geqslant(1-\varepsilon) f$ on $\Omega_{1} \times \Omega_{2}$. The proof of this claim is given by splitting into four cases, according to whether $x_{1}$ is in $\delta_{1}$ or not, and $x_{2}$ is in $\delta_{2}$ or not.

Case (i). Let $\left(x_{1}, x_{2}\right)$ be in $\delta_{1} \times \delta_{2}$. Then from the definition of $u$ and $w$, we have

$$
\begin{aligned}
u\left(x_{1}, x_{2}\right) & =\iint\left(v\left(y_{1}, y_{2}\right)+2 h\left(y_{1}, y_{2}\right)\right) d \rho_{x_{1}}^{\delta_{1}}\left(y_{1}\right) d \rho_{x_{2}}^{\delta_{2}}\left(y_{2}\right) \\
& \geqslant \iint\left(v\left(z_{1}, z_{2}\right)+\varepsilon\right) h\left(y_{1}, y_{2}\right) d \rho_{x_{1}}^{\delta_{1}}\left(y_{1}\right) d \rho_{x_{2}}^{\delta_{2}}\left(y_{2}\right) \\
& =\left(v\left(z_{1}, z_{2}\right)+\varepsilon\right) h\left(x_{1}, x_{2}\right) \quad(\text { as } h \text { is 2-harmonic) } \\
& \left.\geqslant\left(f\left(z_{1}, z_{2}\right)+\varepsilon\right) h\left(x_{1}, x_{2}\right) \quad(3) \text { as } \bar{\delta}_{1} \times \bar{\delta}_{2} \subset U\right) \\
& >f\left(x_{1}, x_{2}\right) \quad(\text { using }(2)) \\
& >(1-\varepsilon) f\left(x_{1}, x_{2}\right) \quad(\text { as } f>0),
\end{aligned}
$$

Case (ii). Let $x_{1}$ be in $\delta_{1}$ and $x_{2}$ not in $\delta_{2}$. Then,

$$
\begin{aligned}
u\left(x_{1}, x_{2}\right) & =\int w\left(y_{1}, x_{2}\right) d \rho_{x_{1}}^{\delta_{1}}\left(y_{1}\right) \\
& =\int\left(v\left(y_{1}, x_{2}\right)+2 \varepsilon h\left(y_{1}, x_{2}\right)\right) d \rho_{x_{1}}^{\delta_{1}}\left(y_{1}\right) \\
& \geqslant \int\left(R f_{y_{1}}\left(x_{2}\right)+2 \varepsilon h\left(y_{1}, x_{2}\right)\right) d \rho_{x_{1}}^{\delta_{1}}\left(y_{1}\right) \\
& \geqslant \int\left(R f_{x_{1}}\left(x_{2}\right)-\varepsilon+2 \varepsilon h\left(y_{1}, x_{2}\right)\right) d \rho_{x_{1}}^{\delta_{1}}\left(y_{1}\right) \quad(\text { using }(4)) \\
& =\left(R f_{x_{1}}\left(x_{2}\right)-\varepsilon\right) \int d \rho_{x_{1}}^{\delta_{1}}\left(y_{1}\right)+2 \varepsilon h\left(x_{1}, x_{2}\right) \\
& >\left(R f_{x_{1}}\left(x_{2}\right)-\varepsilon\right)(1-\varepsilon)+2 \varepsilon h\left(x_{1}, x_{2}\right) \quad(\text { using }(5)) \\
& >\left(f_{x_{1}}\left(x_{2}\right)-\varepsilon\right)(1-\varepsilon)+2 \varepsilon h\left(x_{1}, x_{2}\right) \quad\left(\text { as } R f_{x_{1}}>f_{x_{1}} \text { on } \Omega_{2}\right) \\
& =\left(f\left(x_{1}, x_{2}\right)-\varepsilon\right)(1-\varepsilon)+2 \varepsilon(1-\varepsilon) \quad(\text { using }(1)) \\
& >(1-\varepsilon) f\left(x_{1}, x_{2}\right) \quad(\text { as } f>0) .
\end{aligned}
$$


Case (iii). Let $x_{1}$ not be in $\delta_{1}$ and $x_{2}$ in $\delta_{2}$. The proof is similar to the previous case.

Case (iv). Let $x_{1}$ not be in $\delta_{1}$ and $x_{2}$ not in $\delta_{2}$. Then the proof trivially follows from the definitions of $u, v$ and $w$. Thus, the claim is proved.

Now, $u \geqslant(1-\varepsilon) f$ on $\Omega_{1} \times \Omega_{2}$ gives that $u \geqslant(1-\varepsilon) v$ on $\Omega_{1} \times \Omega_{2}$. In particular, if $\left(x_{1}, x_{2}\right)$ is in $\delta_{1} \times \delta_{2}$ then

$$
(1-\varepsilon) v\left(x_{1}, x_{2}\right) \leqslant \iint v\left(y_{1}, y_{2}\right) d \rho_{x_{1}}^{\delta_{1}}\left(y_{1}\right) d \rho_{x_{2}}^{\delta_{2}}\left(y_{2}\right)+2 h\left(x_{1}, x_{2}\right) .
$$

The right-hand side of the above inequality is a 2-harmonic function on $\delta_{1} \times \delta_{2}$, hence is a continuous function on $\delta_{1} \times \delta_{2}$. Taking limsup as $\left(x_{1}, x_{2}\right) \rightarrow\left(z_{1}, z_{2}\right)$ in $\Omega_{1} \times \Omega_{2}$, and noticing that $v$ is a 2-superharmonic function we get

$$
\begin{gathered}
(1-\varepsilon) \limsup _{\substack{\left(x_{1}, x_{2}\right) \rightarrow\left(z_{1}, z_{2}\right) \\
\left(x_{1}, x_{1}\right) \text { in } \Omega_{1} \times \Omega_{2}}} v\left(x_{1}, x_{2}\right) \\
\leqslant v\left(z_{1}, z_{2}\right)+2 \varepsilon h\left(z_{1}, z_{2}\right) \\
\leqslant v\left(z_{1}, z_{2}\right)+2 \varepsilon .
\end{gathered}
$$

As $\varepsilon>0$ is arbitrary, we have

$$
\limsup _{\left(x_{1}, x_{2}\right) \rightarrow\left(z_{1}, z_{2}\right)} v\left(x_{1}, x_{2}\right) \leqslant v\left(z_{1}, z_{2}\right) \text {. }
$$

Since $\left(z_{1}, z_{2}\right)$ is an arbitrary point in $\Omega_{1} \times \Omega_{2}$, it follows that $v$ is upper semicontinuous on $\Omega_{1} \times \Omega_{2}$. Hence, $v$ is continuous on $\Omega_{1} \times \Omega_{2}$, and this concludes the proof for the case $n=2$.

To complete the proof of the induction, we proceed from the case of functions of $n-1$ variables to functions of $n$ variables in exactly the same way. We remark that the choice of $u$ in the above proof is replaced by $w_{n}$ as defined in Proposition 4 . The rest of the details are absolutely the same. This allows us to conclude that $M R f$ is in general a continuous function whenever $f$ is a nonnegative continuous function with compact support, completing the proof of the theorem.

As an immediate consequence, we have the following corollary.

COROLlaRY 8. If $f$ is a nonnegative continuous function on $\Omega$ with compact support, then MRf is a continuous n-potential on $\Omega$.

Though the following result is essentially a corollary to the above theorem, we will state it as a theorem due to its importance. We omit the proof.

THEOREM 9. Let $v$ be a positive $n$-superharmonic function on $\Omega$. Then, there is a sequence $v_{j}$ of continuous n-potentials such that $v_{j}$ increases pointwise to $v$ on $\Omega$ as $j \rightarrow \infty$.

From now on $K$ is a compact $n$-polar subset of $\Omega$, such that every $i$-section of $K$ through any point of $\Omega$ is $(n-i)$-polar, for $i=1,2, \ldots, n-1$.

The next theorem is the converse of Theorem 3 .

THEOREM 10. Given a positive continuous function $f$ on $K$ and an $\varepsilon>0$, there exists a continuous n-potential $p$ on $\Omega$ such that $|f-p|<\varepsilon$ on $K$. 
Proof. Let $\left\{U_{j}\right\}, j=1,2,3, \ldots$, be a decreasing sequence of relatively compact subsets of $\Omega$ such that $U_{j} \supset \bar{U}_{j+1} \supset U_{j+1}$ for $j=1,2, \ldots$, and $K=$ $\bigcap\left\{U_{j}: j=1,2,3, \ldots\right\}$. For each $j$, let $f_{j}$ be a nonnegative continuous extension of $f$ to $\Omega$ with support of $f_{j} \subset U_{j}$. By taking infimum at each stage, we may assume that $\left\{f_{j}\right\}$ is a decreasing sequence of functions on $\Omega$.

Put $p_{j}=M R f_{j}$. Then, by Corollary $8, p_{j}$ is a continuous $n$-potential, for each $j$. Following the proof Theorem 4 of [7], we can show that $p_{j}$ decreases pointwise to $f$ on $k$ as $j \rightarrow \infty$. Using Dini's Theorem, we conclude that $p_{j}$ converges to $f$ uniformly on $K$. Thus, there is an $m$ such that $\left|f(x)-p_{j}(x)\right|<\varepsilon$ if $j \geqslant m$, for all $x$ in $K$. The choice $p=p_{m}$ meets the requirement of the theorem, completing the proof.

The following result is an analogue of Theorem 5 of [7], and can be proved analogously with the help of Theorem 10 .

Proposition 11. Let $f_{0}$ be a positive continuous function on $K$, and $F_{0}$ be a relatively compact open neighborhood of $K$. Put $F=\bar{F}_{0}$, and let $f$ be a nonnegative continuous extension of $f_{0}$ to $\Omega$, such that $f>0$ on $F$. Then, given $\varepsilon>0$, there exists $a$ continuous potential $p$ on $\Omega$ such that $p<f$ on $F$, and $p \geqslant f_{0}-\varepsilon$ on $K$.

Our ultimate aim is the following theorem, for the case $n \geqslant 2$.

THEOREM 12. Given a positive continuous function $f_{0}$ on $K$, there is a continuous $n$-potential $p$ on $\Omega$ such that $p=f_{0}$ on $K$.

Proof. The existence of an $n$-potential $p$ such that $p=f_{0}$ on $K$ can be proved as in the case $n=1$. (See Theorem 2 of [7].) However, in proving the continuity of $p$, in the case $n=1$, we have explicitly used the fact that $R g$ is harmonic outside the support of $g$. This result is no longer valid for $M R g$ when $n>1$. Hence, we modify the proof as follows. We also note that the same method works in the case $n=1$.

Let $\varepsilon>0$. For a continuous function $g$ on $K$, define

$$
\|g\|_{K}=\sup \{|g(x)|: x \text { in } K\},
$$

and if $g$ is a bounded continuous function on $\Omega$, then define

$$
\|g\|_{\infty}=\sup \{|g(x)|: x \text { in } \Omega\} .
$$

Let $q$ be a bounded continuous $n$-potential on $\Omega$. We may assume that $q \geqslant 1$ on $K$.

Let $F_{0}$ be a relatively compact open set containing $K$ and let $F=\bar{F}_{0}$. Choose $f$ a nonnegative continuous extension of $f_{0}$ to $\Omega$ with $f>0$ on $F$, and $\|f\|_{\infty}=\left\|f_{0}\right\|_{K}$. Then, by the previous theorem, there is a continuous $n$-potential $q_{0}$ on $\Omega$ such that $q_{0}<f$ on $F$ and $q_{0}>f_{0}-\varepsilon$ on $K$. Let $p_{0}=\inf \left\{\left\|f_{0}\right\|_{K} q, q_{0}\right\}$ on $\Omega$. Then, $p_{0}$ is a bounded continuous $n$-potential on $\Omega$. Further, $p_{0} \leqslant q_{0}<f$ on $F$. If $x$ is in $K$, then $q(x) \geqslant 1$, and hence,

$$
\left\|f_{0}\right\|_{K} q(x) \geqslant\left\|f_{0}\right\|_{K}=\|f\|_{\infty} \geqslant f(x)>q_{0}(x) .
$$


Therefore, $p_{0}=q_{0}$ on $K$ and hence, $p_{0} \geqslant f_{0}-\varepsilon$ on $K$. Thus, there is a bounded continuous $n$-potential $p_{0}$ such that

1. $p_{0}(x)<f(x)$ for all $x$ in $F$,

2. $p_{0}(x) \geqslant f_{0}(x)-\varepsilon$ for all $x$ in $K$,

3. $\left\|p_{0}\right\|_{\infty}<\left\|f_{0}\right\|_{K}\|q\|_{\infty}$.

Put $g_{1}=\max \left(f-p_{0}, 0\right)$ on $\Omega$. Then, $g_{1}$ is a nonnegative continuous function and $g_{1}>0$ on $F$. Let $g_{2}$ be a nonnegative continuous on $\Omega$ such that $g_{2}=g_{1}$ on $K$ and $g_{2}>0$ on $F$. Since $g_{2}=g_{1}=f-p_{0}$ on $K$, we may even choose $g_{2}$ such that $\left\|g_{2}\right\|_{\infty}=\left\|f-p_{0}\right\|_{K}$. Set $f_{1}=\inf \left\{g_{1}, g_{2}\right\}$. Then, $f_{1}$ is a nonnegative continuous function on $\Omega$ with $f_{1}>0$ on $F$ and $f_{1}=f-p_{0}$ on $K$. As before, there is a continuous $n$-potential $p_{1}$ such that

1. $p_{1}(x)<f_{1}(x)$ for every $x$ in $F$,

2. $p_{1}(x) \geqslant f_{1}(x)-\varepsilon / 2$ for every $x$ in $K$,

3. $\left\|p_{1}\right\|_{\infty} \leqslant\left\|f_{1}\right\|_{K}\|q\|_{\infty}$.

Now, $f_{1}(x) \leqslant g_{1}(x) \leqslant f(x)-p_{0}(x)$ on $F$, and $f_{1}(x)=g_{1}(x)=f(x)-p_{0}(x)$ on $K$.

Therefore, the above inequalities can be rewritten as follows.

1. $p_{0}(x)+p_{1}(x)<f(x)$ for all $x$ in $F$,

2. $p_{0}(x)+p_{1}(x) \geqslant f_{0}(x)-\varepsilon / 2$ for all $x$ in $K$,

3. $\left\|p_{0}\right\|_{\infty} \leqslant\left\|f_{0}\right\|_{K}\|q\|_{\infty}$ and $\left\|p_{1}\right\|_{\infty} \leqslant\left\|f_{1}\right\|_{K}\|q\|_{\infty}$.

Note that $\left\|f_{1}\right\|_{K} \leqslant \varepsilon / 2$.

Proceeding by induction, we get the sequence $\left\{p_{m}\right\}, m=0,1,2, \ldots$, of bounded continuous $n$-potentials and a sequence $\left\{f_{m}\right\}, m=0,1,2, \ldots$, of continuous functions such that

1. $\sum_{i=0}^{m} p_{i}<f$ on $F$ for every $m$,

2. $\sum_{i=0}^{m} p_{i}>f_{0}-\varepsilon / 2^{m}$ on $K$ for every $m$,

3. $\left\|p_{m}\right\|_{\infty} \leqslant\left\|f_{m}\right\|_{K}\|q\|_{\infty}$ for $m=0,1,2, \ldots$

Note that $\left\|f_{m}\right\|_{K}<\varepsilon / 2^{m}$ for $m=1,2,3, \ldots$

Set $p=\sum_{i=0}^{\infty} p_{m}$ on $\Omega$. Then, it is clear that $p$ is an $n$-superharmonic function and that $p \leqslant f$ on $F$ and $p=f_{0}$ on $K$. By an analogue of Proposition 2.2.2 of [2], $p$ is an $n$-potential on $\Omega$. As $\left\|p_{m}\right\|_{\infty} \leqslant\left\|f_{m}\right\|_{K}\|q\|_{\infty} \leqslant\left(\varepsilon / 2^{m}\right)\|q\|_{\infty}$ for $m \geqslant 1, \sum_{m=0}^{\infty} p_{m}(x)$ converges uniformly on $\Omega$. Since each $p_{m}$ is a continuous function, $p$ is continuous on $\Omega$, completing the proof.

The following corollary is an immediate consequence of the above theorem.

COROLlaRY 13. (1) Every real-valued continuous function on $K$ is the restriction to $K$ of the difference of two positive continuous n-potentials on $\Omega$.

(2) Every positive lower semicontinuous function on $K$ is the restriction to $K$ of an n-potential.

(3) If the constant function 1 is $n$-superharmonic on $\Omega$, then every real-valued continuous function on $K$ is the restriction to $K$ of an $n$-superharmonic function on $\Omega$.

\section{BIBLIOGRAPHY}

1. M. Brelot, Lectures on potential theory, Tata Institute of Fundamental Research, Bombay, 1960, re-issued 1967.

2. C. Constantinescu and A. Cornea, Potential theory on harmonic spaces, Springer-Verlag, 1972. 
3. K. Gowrisankaran, Multiply harmonic functions, Nagoya Math. J. 28 (1966), 27-48.

4. __ Multiply superharmonic functions, Ann. Inst. Fourier (Grenoble) 4 (1975), 235-244.

5. R. M. Herve, Recherches axiomatiques sur la théorie de fonctions surharmoniques et du potential, Ann. Inst. Fourier (Grenoble) 12 (1962), 415-571.

6. R. Jesuraj, Continuous functions and exceptional sets in potential theory, Ph.D. Thesis, McGill Univ., Montreal, Quebec, 1981.

7. Continuous functions on polar sets, Proc. Amer. Math. Soc. 93 (1985), 262-266.

8. D. Singman, Exceptional sets in a product of harmonic spaces, Math. Ann. 262 (1983), 29-43.

Prime Computers, 500 Old Connecticut Path, Framingham, Massachusetts 01701

Current address: Digital Equipment Corporation, 550 King Street, LKG1-3/A06, Littleton, Massachusetts $01460-1289$ 